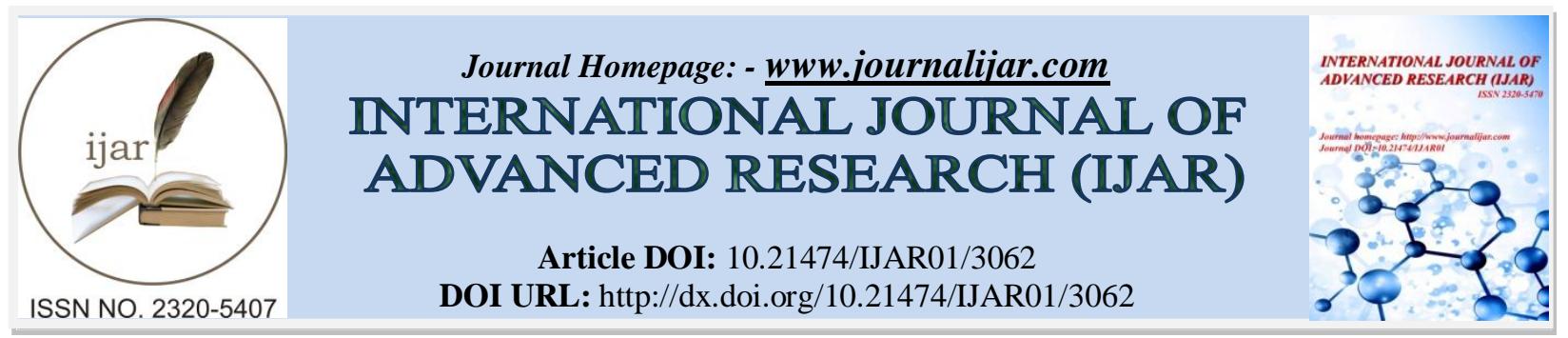

RESEARCH ARTICLE

\title{
BIOLOGIC AND MORPHOLOGIC MARKERS OF THE THR OMEMBOLIC RISK IN THE ATRIAL FIBRILLATION
}

H. Jallal, A. Zbitou, S. Arioua, L. Bendriss and a. Khatouri. Service de cardiologie Hopital Militaire Avicenne. CHU Mohammed VI Marrakech.

\section{Manuscript Info}

\section{Manuscript History}

Received: 01 December 2016

Final Accepted: 29 December 2016

Published: January 2017

\section{Abstract}

Atrial fibrillation (AF) is the most common arrhythmia, In certain case he can expose to systemic thrombo-embolic complications. Antithrombotic treatment, with anticoagulant or antiplatelet agents is guided by the evaluation of the ischaemic and haemorrhagic risk based on validated clinical risk scores. However, these scores have limitations and their diagnostic performances are variable, which limit their use in daily practice; Many biomarkers and morphologic markers have been Studied to refine the stratification of thromboembolic risk, and should be able to predict a more focused and more rational prescription of anticoagulant treatment.

Copy Right, IJAR, 2016,. All rights reserved.

\section{Introduction:-}

La fibrillation auriculaire est l'arythmie la plus fréquente en pratique clinique associée à une augmentation significative de la morbi-mortalité cardiovasculaire. Son incidence et sa prévalence augmentent avec l'âge, faisant de cette pathologie une véritable épidémie avec un fort impact économique. Il existe au cours de la FA un état prothombotique, se caractérisant par un taux sanguin élevé de différentes protéines procoagulant, mais également pouvant être marqués par des marqueurs morphologiques à l'échocardiographie. Les scores cliniques CHADS2et CHA2DS2-VASc sont simples et pratiques en utilisation quotidienne, et la question se pose de savoir si l'on peut améliorer cette approche par la prise en compte des ces biomarqueurs ou de données échographiques (« échomarqueurs »).

\section{Mecanisme de la thrombogenese dans la fa:-}

La pathogénie de la thrombose au cours de la FA ne relève pas seulement de la stase sanguine au niveau de l'auricule gauche. La FA serait en elle-même un état d'hypercoagulabilité et obéirait à la triade de Virchow : stase sanguine (perte de la systole auriculaire), altérations de la paroi vasculaire (documentées notamment par des études en microscopie électronique des parois auriculaires) et anomalies de la crase sanguine. Un état d'hypercoagulabilité correspondant à cette troisième composante a été identifié dans l'HTA, la pathologie coronaire, vasculaire cérébrale et le diabète. Plusieurs études ont suggéré le rôle d'une composante inflammatoire dans l'initiation et la pérennisation de la FA, d'autres travaux ont permis de soupçonner un lien entre l'inflammation et la thrombose.

Les mécanismes physiopathologiques ont évolué depuis la classique triade de Virchow décrite en 1855 ce qui a amener a une révision de cette dernière comme le montre ce schéma ci-dessous :

Corresponding Author:- H. Jallal.

Address:- Service de cardiologie Hopital Militaire Avicenne. CHU Mohammed VI marrakech. 


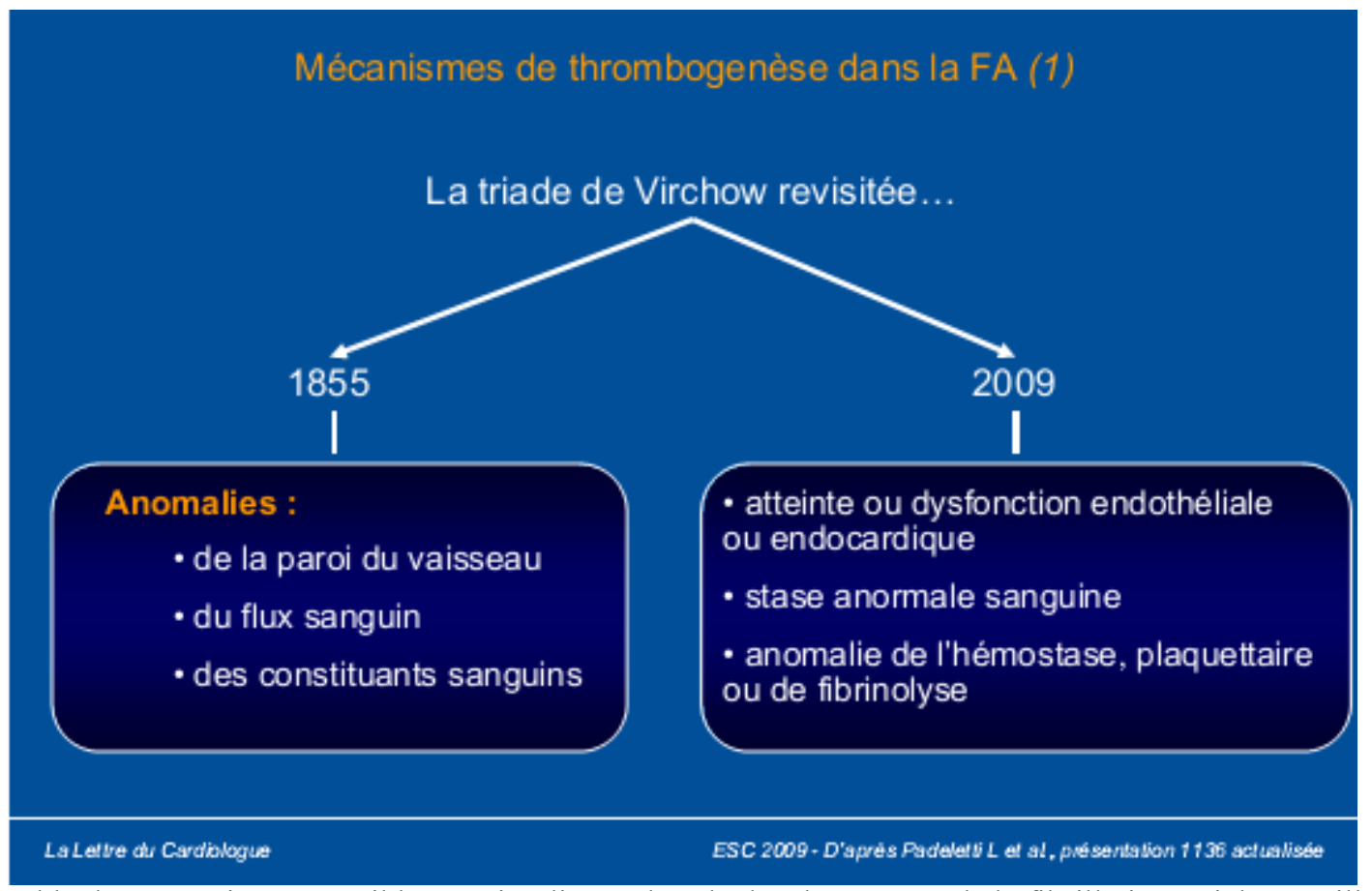

L'ensemble des mécanismes possiblement impliqués dans le développement de la fibrillation atriale sont illustrés dans le schéma suivant :

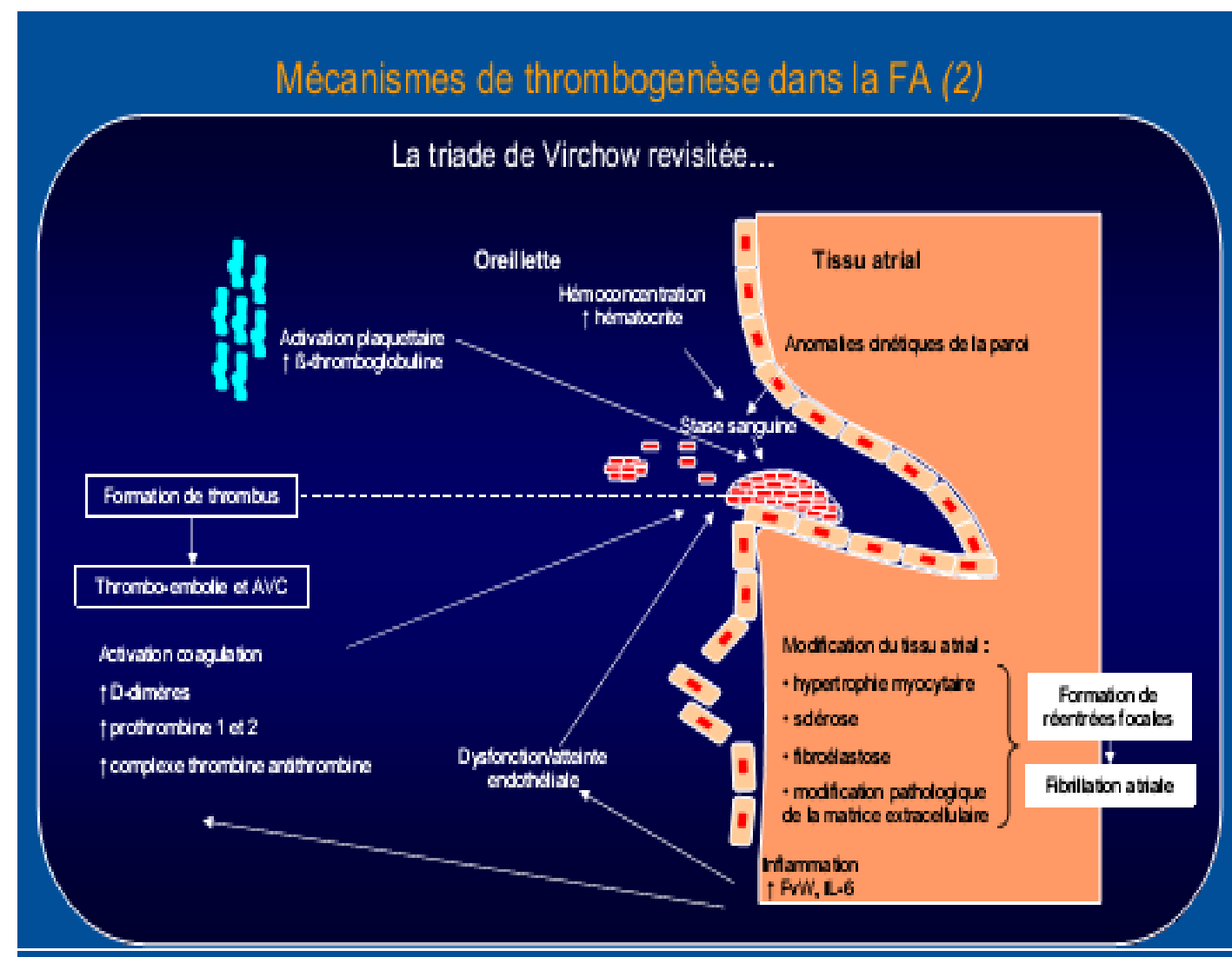




\section{Score de risque et risque embolique arteriel dans la fa:-}

De nombreuses approches ont successivement été proposées au cours des 20 dernières années, basées sur des études épidémiologiques ou des revues de la littérature, ayant intégré plusieurs facteurs de risque, mais à des degrés variables : âge, sexe féminin, antécédent d'infarctus cérébral ou AIT, hypertension artérielle, insuffisance cardiaque, diabète et pathologie vasculaire, cette dernière dernièrement intégrée dans le score CHA2DS2-VASc.

Cependant, ces scores sont imparfaits car issus de cohortes évaluant certes le risque embolique lié à ces facteurs, mais moins de $10 \%$ des patients triés et éligibles ont été inclus dans les essais, qui ont par ailleurs exclu de nombreux patients ayant une contre-indication au traitement anticoagulant, ce qui explique que certains facteurs de risque n'ont pas été pris en considération. Ainsi, l'extrapolation de ces données est difficile à envisager dans le monde réel.

Faut-il calculer le score C-statistique pour déterminer le meilleur score, prédicteur des événements ischémiques, ou faut-il au contraire proposer une reclassification des patients, puisqu'il a été indiqué que le score $\mathrm{C}$-statistique validé dans une étude ne peut être comparé à une autre étude, du fait de l'hétérogénéité des populations incluses et du profil de risque embolique particulier [1], De plus, la prescription d'un traitement anticoagulant oral, basé sur des recommandations consensuelles, ne correspond pas à ce qui est réellement observé, puisque la proportion de patients sous anticoagulant est souvent indépendante de la stratification du risque en faible, intermédiaire ou élevé [2]. Ces différentes considérations doivent également être mises en perspective avec d'autres observations telles que, en particulier, la diminution du taux de prescription de l'aspirine chez les patients en FA.

Les scores cliniques CHADS2et CHA2DS2-VASc sont simples et pratiques en utilisation quotidienne, et la question se pose de savoir si l'on peut améliorer cette approche par la prise en compte des biomarqueurs ou de données échographiques (« échomarqueurs »).

\section{Marqueurs bilogiques de l'hemostase:-}

Le dosage de marqueurs plasmatiques explore spécifiquement chacune des étapes de l'hémostase. Un état d'hypercoagulabilité peut concerner l'hémostase primaire (lésion ou dysfonction endothéliale, activation plaquettaire augmentée), la cascade de la coagulation (activité thrombine, fibrinoformation augmentées) ou la fibrinolyse (hypofibrinolyse) (tableau1)

Tableau 1:- Marqueurs de la coagulation (dosages, abréviations, métabolisme)

\begin{tabular}{|c|c|c|c|}
\hline & Marqueurs (unités de dosage) & Abréviations & Métabolisme \\
\hline \multirow[t]{5}{*}{ Plaquettes } & Facteur plaquettaire 4 ( $\mathrm{ng} / \mathrm{mL})$ & PF4 & Chimiokine stockée dans les granules alpha \\
\hline & Bêta-thromboglobuline (UVmL) & BTG & Chimiokine stockée dans les granules alpha \\
\hline & $\begin{array}{l}\text { P-sélectine soluble }(\mathrm{ng} / \mathrm{mL}) \\
\text { et membranaire (CD } 62 \mathrm{P})\end{array}$ & $\begin{array}{l}\text { sP-Sel } \\
\text { mP-Sel }\end{array}$ & $\begin{array}{l}\text { Protéine transmembranaire des granules plaquettaires (et } \\
\text { endothéliaux) }\end{array}$ \\
\hline & (\%/plaquette) & & $\begin{array}{l}\text { Expression sur } \mathbf{l} \text { membrane cytosolique, interactions avec } \\
\text { leucocytes et c. endoth libles }\end{array}$ \\
\hline & $\begin{array}{l}\text { Glycoproteine V soluble } \\
(n g / \mathrm{mL})\end{array}$ & sGPV & $\begin{array}{l}\text { Protèine transmembranaire cytosolique } \\
\text { Fragment soluble libéré après hydrolyse par la thrombine }\end{array}$ \\
\hline \multirow[t]{2}{*}{ Endothélium } & Facteur won Willebrand (UI/dL) & $\mathrm{Wb}$ & $\begin{array}{l}\text { Glycoproténe stockée dans les granules endothéliaux (et } \\
\text { plaquettaires) } \\
\text { Liaison au sous-endothélium et à la GPIb } \\
\text { Liaison plasmatique au VIII }\end{array}$ \\
\hline & Thrombomoduline $(\mathrm{ng} / \mathrm{mL})$ & STM & $\begin{array}{l}\text { Protéine transmembranaire cytosolique } \\
\text { Liaison à la thrombine pour activer la proteine C }\end{array}$ \\
\hline \multirow[t]{3}{*}{$\begin{array}{l}\text { Activite } \\
\text { thrombine }\end{array}$} & $\begin{array}{l}\text { Fragments I et } 2 \\
\text { de la prothrombine (nmollL) }\end{array}$ & $\mathrm{P} \mid+2$ & $\begin{array}{l}\text { Sous-unités de la prothrombine libérées lors de son hydrolyse en } \\
\text { thrombine }\end{array}$ \\
\hline & $\begin{array}{l}\text { Complexes thrombine- } \\
\text { antithrombine III ( } \mathrm{ng} / \mathrm{mL})\end{array}$ & TAT III & $\begin{array}{l}\text { Complexes formés par la liaison de b thrombine à son inhibiteur, } \\
\text { TAT III }\end{array}$ \\
\hline & Fibrinopeptide A (ng/mL) & $\mathrm{FpA}$ & Peptide issu du divage du fibrinogène par la thrombine \\
\hline \multirow[t]{2}{*}{ Fibrinoformation } & Fibrinogène $(g / l)$ & $\mathrm{Fb}$ & Précurseur de $\mathbf{b}$ fibrine \\
\hline & D-dimères $(\mathrm{ng} / \mathrm{mL})$ & Ddi & $\begin{array}{l}\text { Produits de dégradation de la fibrine stabilisée par le facteur XIII } \\
\text { Reflet du turn over de la fibrine }\end{array}$ \\
\hline \multirow[t]{3}{*}{ Fibrinalyse } & $\mathrm{t}-\mathrm{PA}(\mathrm{ng} / \mathrm{mL})$ & - & $\begin{array}{l}\text { Protéine circulante sécrétée par les cellules endoth eliales } \\
\text { Clive le plasminogène en plasmine }\end{array}$ \\
\hline & PALI (UVdL) & - & Inhibiteur du t-PA \\
\hline & Complexes plasmine-alpha2-antiplasmine ( $\mathrm{ng} / \mathrm{mL}$ ) & PAP & Complexes formés par la liaison de la plasmine à son inhibiteur \\
\hline
\end{tabular}


Plusieurs études ont montré l'intérêt de ces biomarqueurs pour confirmer les scores cliniques [3-4]. Il s'agit de marqueurs d'hypercoagulation, d'inflammation, d'activation plaquettaire ou endothéliale, de facteur Willebrand, d'index d'atteinte endothéliale. Les plaquettes sont également activées dans la FA, mais l'activation plaquettaire pourrait ne refléter que l'association entre comorbidité et FA (par exemple, HTA et pathologie vasculaire) plutôt qu'être liée à l'état prothrombotique dans la FA [5]. Les concentrations du facteur Willebrand plasmatique constituent un facteur de risque indépendant d'événements cardiovasculaires, incluant la mortalité et le saignement majeur chez des patients sous anticoagulants du fait d'une FA permanente [5]. L'élévation des D-dimères est associée à un excès de risque chez les patients en FA, Plusieurs travaux [6-7] se sont intéressés à l'évaluation de la valeur prédictive des d-dimères sur le risque de survenue d'un événement thromboembolique. Nozawa et al. [6] ont constaté que le risque thromboembolique chez les patients à faible risque clinique d'accident thromboembolique était faible quand le taux de d-dimères était inférieur à $150 \mathrm{ng} / \mathrm{mL}(0,7 \% / \mathrm{an})$. Ce risque n'était plus négligeable lorsque le taux de d-dimères était supérieur à $150 \mathrm{ng} / \mathrm{mL}(3,8 \% / \mathrm{an})$.

L'élévation de la CRP est associée à un excès de risque de décès, mais pas à un risque accru d'infarctus cérébral chez des patients en FA, confirmant l'intérêt de la CRP chez des patients ayant une pathologie vasculaire. Lip (8) a montré que le facteur Willebrand plasmatique permettait d'améliorer la prédiction du score CHADS2 en ce qui concerne celle du risque d'AIC et d'AVC chez les patients en FA inclus dans l'étude SPAF-III. Plus récemment, la troponine et le BNP ont été validés comme associés à un surrisque d'événements dans la population RE-LY traitée par anticoagulants [9].

La fonction rénale, la clairance de la créatinine, la protéinurie, le débit de filtration glomérulaire ont également été considérés comme associés à un excès de risque d'événement cardiovasculaire, L'insuffisance rénale est associée au risque d'insuffisance cardiaque et d'HTA, augmente avec l'âge, la présence d'un diabète ou la pathologie vasculaire, tous composants des scores CHADS2et CHA2DS2-VASc. En attendant des validations, le score CHA2DS2-VASc pourrait être implémenté par la prise en compte de un ou deux points supplémentaires en cas d'insuffisance rénale. Deux publications récentes ont permis de montrer qu'un score R2-CHADS2 ou R-CHA2DS2-VASc [10] pouvait améliorer la prédiction des événements cardiovasculaires chez ces patients.

\section{Marqueurs morhologiques ou echomarqeurs:-}

L'échocardiographie est une technique largement utilisée et versatile et qui peut apporter des informations complémentaires dans la stratification du risque des patients en FA, Cependant, la préconisation de la réalisation de cet examen chez tous les patients en FA pourrait accroître la complexité d'évaluation du risque thromboembolique.

\section{'échographie transthoracique:-}

Une fraction d'éjection ventriculaire gauche (FEVG) normale est associée à l'absence de formation de thrombus dans l'auricule gauche [11]. L'évaluation de la FEVG améliore la capacité de discrimination dans l'utilisation des scores CHADS2et CHA2DS2-VASc en ce qui concerne la détection d'un thrombus atrial gauche [12] et d'autres marqueurs de la stase atriale gauche.

La masse VG indexée aurait une valeur discriminante intéressante pour prédire les thrombi dans l'auricule gauche avec un score C-statistique à 0,98 , bien plus élevé que pour le contraste spontané $(0,73-0,75)$ [13]. Dans l'étude SPAF [14], la taille de l'OG mesurée en mode $M$ était un prédicteur indépendant du risque thromboembolique et améliorait la valeur discriminante de la seule approche clinique, La surface atriale gauche et la FEVG augmentent la capacité de discrimination des scores CHADS et CHADS-VASc en ce qui concerne la détection des thrombi dans l'auricule gauche, le contraste spontané intense et les basses vélocités auriculaires gauches mais La combinaison de la taille de l'OG et de la FEVG a été proposée comme approche ayant une bonne capacité de discrimination des patients sans thrombus auriculaire gauche à l'ETO. Un rapport FEVG/volume atrial gauche indexé supérieur à 1,5 exclut la présence d'un thrombus dans l'auricule gauche chez les patients en FA, candidats à une ablation ou à une cardioversion [12].

Le strain longitudinal pourrait constituer une information indépendamment du volume de l'oreillette gauche indexée. Le volume atrial gauche est une mesure statique, alors que le strain longitudinal est un paramètre dynamique qui reflète les variations de dimension de l'oreillette gauche en phase de remplissage. Ce caractère dynamique des variations de dimensions de l'oreillette gauche pourrait influencer la stase atriale gauche, le temps de transit sanguin sur la surface endocardique, et donc la plus ou moins grande propension à thromboser. Une réduction 
du strain longitudinal atrial gauche a été associée à la présence d'une fibrose myocardique [90], et ainsi la fibrose atriale gauche, détectée par IRM, a été associée au risque d'infarctus cérébral chez les patients en FA [91].

\section{'échographie transoesophagienne:-}

La plupart des études qui se sont intéressées aux données d'échocardiographie transœsophagienne (ETO) montrent que la présence d'un contraste spontané, de basses vélocités auriculaires gauches, d'un thrombus atrial gauche ou l'existence d'athérome aortique complexe de l'aorte descendante seraient des prédicteurs indépendants du risque embolique artériel. Dans l'étude de Zabalgoitia M [16], l'âge, les vélocités auriculaires gauches, le diamètre atrial gauche mesuré en mode $\mathrm{M}$, la présence d'un athérome aortique et le taux de fibrinogène plasmatique sont associés de façon indépendante à la présence d'un contraste spontané atrial gauche, tandis que des vélocités auriculaires gauches antérogrades, $<20 \mathrm{~cm} / \mathrm{s}$, et des facteurs de risque clinique sont associés de façon indépendante à la présence d'un thrombus atrial ou auriculaire gauche [95]. Une étude ayant inclus 500 patients avec infarctus cérébral en rythme sinusal ou en FA [96] a montré que le risque de thrombose dans l'auricule gauche, avec présence de contraste spontané, augmentait de façon significative lorsque la vélocité auriculaire gauche était inférieure à 57 $\mathrm{cm} / \mathrm{s}$. Lorsqu'elle est $>$ ? $55 \mathrm{~cm} / \mathrm{s}$, le risque de thrombose atrial gauche est minime, de même que le risque de contraste spontané est très faible avec une valeur prédictive négative respectivement de 99 et $100 \%$.En analyse multivariée, la vélocité auriculaire gauche est le meilleur prédicteur de l'apparition d'un thrombus ou d'un contraste spontané.

\section{corrélation entre les marqueurs biologiques et morphologiques:-}

Heppellet al. [17] et Pongratzet al. [18] ont trouvé des marqueurs d'activation plaquettaire (BTG, P-sélectine et PF4) significativement plus élevés en présence de contraste spontané ou d'un thrombus et Heppellet al. un facteur de Willebrand plus élevé en présence d'un thrombus. Pour les marqueurs de la coagulation, les résultats sont divergents, sans explication claire : en présence d'un thrombus, complexes TAT III et D-dimères plus élevés pour Heppellet al. [17], non significativement élevés pour Pongratzet al.[36]. Un travail préliminaire de Somloiet al. [19] chez 73 patients bénéficiant d'une ETO avant cardioversion, a rapporté une valeur prédictive négative des Ddimères de $98 \%$, pour éliminer la présence d'un thrombus auriculaire gauche. Nakagawaet al.[20] ont trouvé, en présence d'un contraste spontané aortique, des D-dimères, des complexes TAT III et complexes plasmine-a2antiplasmine (PAP) significativement plus élevés. On ne dispose pas de recherche de corrélation entre les marqueurs biologiques et l'athérome aortique

Les données échographiques pourraient donc apporter une précision supplémentaire pour évaluer le risque thromboembolique artériel, une perspective d'intégration de ces échomarqeurs en plus des biomarqueurs serait intéressante à envisager dans le contexte de la FA (Fig. 1). 


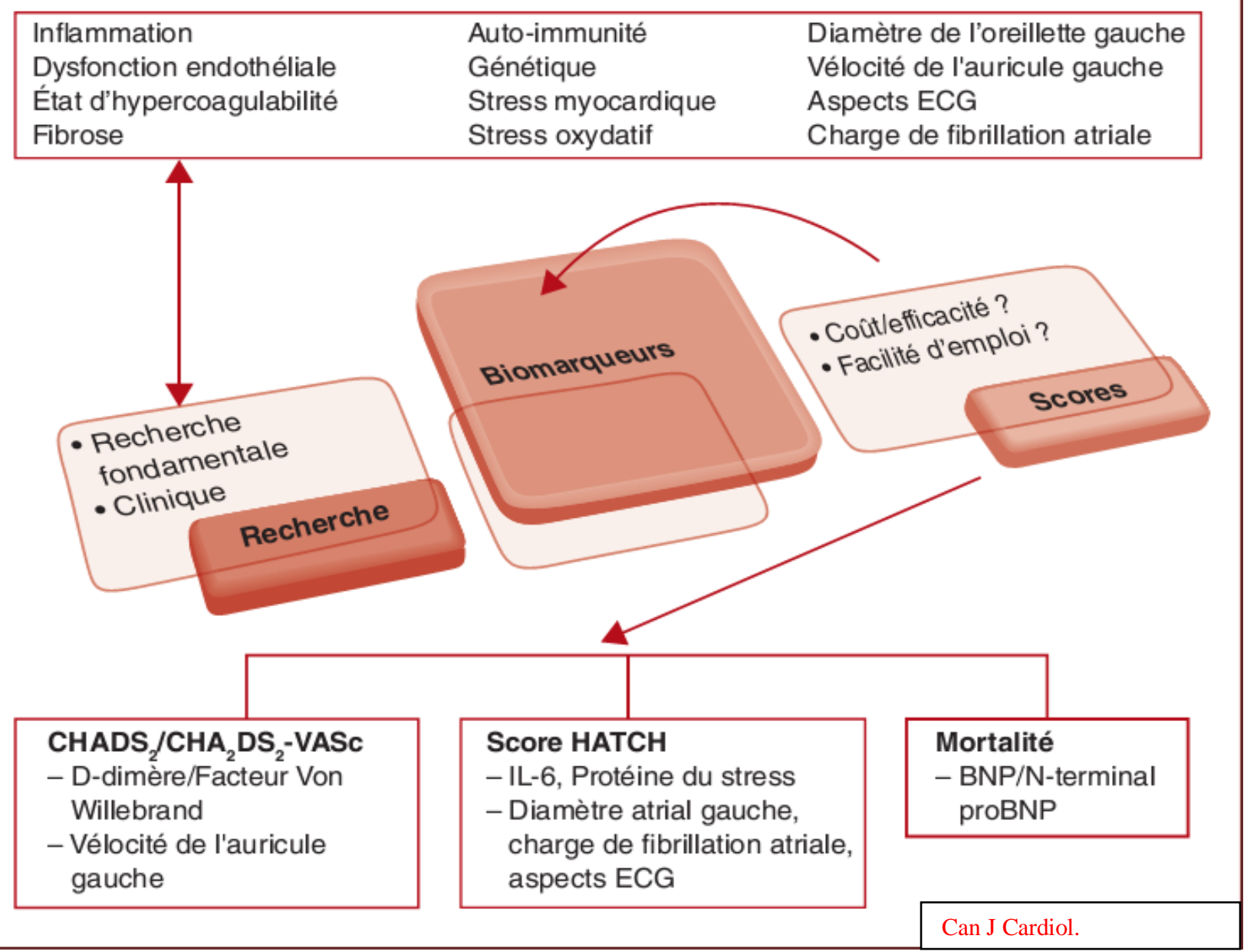

Schéma intégrant les différentes approches clinique, biologique et morphologique

\section{Conclusion:-}

La prise en charge thérapeutique de la FA s'avère souvent difficile. Cependant, les dernières années ont été marquées par un progrès dans l'évaluation du risque embolique artériel et hémorragique, ainsi que les possibilités thérapeutiques des antivitamines $\mathrm{K}$ et des anticoagulants oraux directs (anti-IIa et anti-Xa), ce progrès a permis d'améliorer la prévention du risque d'infarctus cérébral, en particulier chez les patients ayant au moins un facteur de risque. Les approches complémentaires morphologiques et biologiques demandent à être validées afin de contribuer à une meilleure stratification du risque et peut-être aboutir à une aide au traitement, surtout chez les patients jugés a tes faible risque ou ceux ayant un risque hémorragique accru.

\section{Reference:-}

1. Fang MC, Go AS, Chang $\mathrm{Y}$ et al (2008). Comparison of risk stratiÀcation schemes to predict thromboembolism in people with nonvalvular atrial fibrillation. J Am Coll Cardiol 2008;51:810-5

2. Wilke T, Groth A, et al (2012). Oral anticoagulation use by patients with atrial fibrillation in Germany. Adherence to guidelines, causes of anticoagulation under-use and its clinical outcomes, based on claims-data of 183,448 patients. Thromb Haemost 2012;107:1053-65.

3. Sadanaga T, Kohsaka S, Ogawa S (2010). D-dimer levels in combina-tion with clinical risk factors can effectively predict subsequent thromboembolic events in patients with atrial fibrillation during oral anticoagulant therapy. Cardiology 2010;117:31-6.i

4. Roldan V, Marin F, Garcia-Herola A, Lip GY(2005). Correlation of plasma von Willebrand factor levels, an index of endothelial damage/dysfunction, with two point-based stroke risk stratifcation scores in atrial Fibrillation. Thromb Res 2005;116:321-5 
5. Watson T, Shantsila E, Lip GY(2005). Mechanisms of thrombogenesis in atrial fibrillation: Virchow's triad revisited. Lancet 2009;373:155-66.

6. Nozawa $\mathrm{T}$, Inoue $\mathrm{H}$, Hirai $\mathrm{T}$, et al(2006). d-dimer level influences thromboembolic events in patients with atrial fibrillation. Int J Cardiol 2006;109:59-65

7. Sadanaga T, Sadanaga M, Ogawa S(2010). Evidence that d-dimer levels predict subsequent thromboembolic and cardiovascular events in patients withatrial fibrillation during oral anticoagulant therapy. J Am Coll Cardiol 2010;55:2225-31

8. Lip GY, Patel JV, Hughes E, Hart RG(2007). High-sensitivity c-reactive protein and soluble CD40 ligand as indices of infflammation and platelet activation in 880 patients with non valvular atrial fibrillation: relationship to stroke risk factors, stroke risk stratification schema, and prognosis. Stroke 2007;38:1229-37.

9. Hijazi Z, Oldgren J, Andersson U, et al(2012). Cardiac biomarkers are associated with an increased risk of stroke and death in patients with atrial fibrillation: a randomized evaluation of long-term anticoagulation therapy (RE-LY) substudy. Circulation 2012;125:1605-16.

10. Barra S, Almeida I, Caetano F, et al(2013). Stroke prediction with an adjusted R-CHA2DS2VASc score in a cohort of patients with a Myocardial Infarction. Thromb Res 2013;132:293-9.

11. Barra S, Almeida I, Caetano F et al(2013). Stroke prediction with an adjusted R-CHA2DS2VASc score in a cohort of patients with a Myocardial Infarction. Thromb Res 2013;132:293-9

12. Kleemann $\mathrm{T}$, Becker $\mathrm{T}$, et al(2009). Prevalence and clinical impact of left atrial thrombus and dense spontaneous echo contrast in patients with atrial fibrillation and low CHADS2 score. Eur J Echocardiogr 2009;10:383

13. Boyd AC, McKay T, Nasibi S,et al(2013). Left ventricular mass predicts left atrial appendage thrombus in persistent atrial fibrillation. Eur Heart J Cardiovasc Imaging 2013;14:269-75.

14. The Stroke Prévention in Atrial Fibrillation Investigators. Predictors of thromboembolism in atrial fibrillation: II. Echocardiographic features of patients at risk. Ann Intern Med 1992;116:6-12

15. Daccarett M, Badger T et al(2011). Association of LA fibrosis detected by delayed-enhancement magnetic resonance imaging and the risk of stroke in patients with atrial fibrillation. J Am Coll Cardiol 2011;57:831-8.

16. Zabalgoitia M, Halperin JL, Pearce L et al(1998). Transesophageal echocardiographic correlates of clinical risk of thromboembolism in nonvalvular atrial fibrillation. Stroke prevention in Atrial Fibrillation III Investigators. J Am Coll Cardiol 1998;31:1622-6.

17. Heppell RM, Berkin KE, Mc Lenachan et al(1997). Haemostatic and haemodynamic abnormalities associated with left atrial thrombosis in non-rheumatic atrial fibrillation. Heart 1997 ; $77: 407-11$.

18. Pongratz G, Brandt-Pohlmann M, Henneke KH, et al(1997). Platelet activation in embolic and preembolic status of patients with nonrheu-matic atrial fibrillation. Chest1997; $111: 929-33$

19. Somloi M, Tomcsanyi J, Nagy E, et al(2003). D-dimer determination as a screening tool to exclude atrial thrombi in atrial fibrillation. Am J Cardiol2003; $92: 85-7$.

20. Nakagawa K, Hirai T, Shinokawa N, et al(2002). Aortic spontaneous echocardiographic contrast and hemostatic markers in patients with non rheumatic atrial fibrillation. Chest2002; $121: 500-5$ 